\title{
Integrated design of discrete-time controller for an active suspension system
}

\author{
Dina Shona Laila \\ Department of Electrical and Electronic Engineering, \\ The University of Melbourne, Parkville 3010, Victoria, \\ Australia. \\ d.laila@pgrad.unimelb.edu.au
}

\begin{abstract}
A novel approach to solve a stabilization problem of an active suspension system using a quarter car model is presented. We apply a combination of our results for the framework of the approximate based direct discrete-time design and the Euler based discrete-time backstepping technique. This stabilization problem is very interesting since utilizing a simple quadratic Lyapunov function brings the system into a LaSalle type stability, which makes the design more complicated. To handle this problem, we use our result on changing supply rates lemma for LaSalle type stability condition, to construct a composite Lyapunov function that can be used for the design within our framework.
\end{abstract}

\section{INTRODUCTION}

Most control systems nowadays are sampled-data in nature. A controller is usually implemented digitally and it is inter-connected with a continuous-time plant via ADC and DAC. In this paper, we study the problem of stabilizing an active suspension system, which is used to enable a car to run smoothly on a rough road for comfortable driving. Presently, active suspension systems are controlled using a hydraulic controller. In view of space limitation in a vehicle, it is most appropriate to use digital device to control the active suspension system, as it requires much less space. Since the active suspension module itself is a mechanical - therefore analog - plant, designing a digital controller for this system is a sampled-data system design.

Recently, a general unifed framework for controller design based on approximate discrete-time models was presented in [10] and further generalized in [7] for the input to state stabilization problem. In particular, the results provide suffcient conditions for the continuous-time plant model, the controller and the approximate discrete-time model, to guarantee that the controller input-to-state stabilizes the exact discrete-time plant model, provided it stabilizes the approximate discretetime plant model.

We design a discrete-time controller to asymptotically stabilize the active suspension system, using the Euler based backstepping technique [9]. Backstepping is a popular technique in nonlinear control design (see [4]). It is then shown that the Euler based discrete-time controller outperforms the emulation controller. This active suspension design problem is very interesting and motivating since the system enjoys a LaSalle type stability when using a simple quadratic Lyapunov function. In [3], where continuous-time stabilization for the same system was considered, stability analysis was done using LaSalle's invariance principle. Unfortunately,
LaSalle's invariance principle is in most cases not applicable when approximate based discrete-time design is used, since semiglobal type of stability is usually achieved. In this situation, we apply our result from [6] to construct a composite Lyapunov function that can be used to characterize stability property of the system.

\section{Preliminaries}

The set of real and natural numbers (including 0) are denoted respectively by $\mathbb{R}$ and $\mathbb{N}$. $\mathcal{S N}$ denotes the class of smooth nondecreasing functions $q: \mathbb{R}_{\geq 0} \rightarrow \mathbb{R}_{\geq 0}$, which satisfy $q(t)>0$ for all $t>0$. A function $\gamma: \mathbb{R}_{\geq 0} \rightarrow \mathbb{R}_{\geq 0}$ is of class $\mathcal{G}$ if it is continuous, nondecreasing and zero at zero. It is of class $\mathcal{K}$ if it is of class $\mathcal{G}$ and strictly increasing; and it is of class $\mathcal{K}_{\infty}$ if it is of class $\mathcal{K}$ and unbounded. Functions of class $\mathcal{K}_{\infty}$ are invertible. A function $\beta: \mathbb{R}_{>0} \times \mathbb{R}_{>0} \rightarrow \mathbb{R}_{>0}$ is of class $\mathcal{K} \mathcal{L}$ if $\beta(\cdot, t)$ is of class $\mathcal{K}$ for each $t \geq 0$ and $\beta(s, \cdot)$ is decreasing to zero for each $s>0$. Given two functions $\alpha(\cdot)$ and $\gamma(\cdot)$, we denote their composition and multiplication respectively as $\alpha \circ \gamma(\cdot)$ and $\alpha(\cdot) \cdot \gamma(\cdot)$.

We consider a parameterized family of discrete-time nonlinear systems of the following form:

$$
x(k+1)=F_{T}(x(k), u(k)), \quad y(k)=h(x(k)),
$$

where $x \in \mathbb{R}^{n}, u \in \mathbb{R}^{m}, y \in \mathbb{R}^{l}$ are respectively the state, input and output of the system. Note that the input $u$ can be a control signal or an exogenous disturbance. It is assumed that $F_{T}$ is well defned for all $x, u$ and suffciently small $T, F_{T}(0,0)=0$ for all $T$ for which $F_{T}$ is de£ned, $h(0)=$ 0 and $F_{T}$ and $h$ are continuous. $T>0$ is the sampling period, which parameterizes the system and can be arbitrarily assigned. The following de£nitions are used to state results presented later in this section.

Defnition 2.1: The system (1) is semiglobally practically input-output to state stable (SP-IOSS), if there exist functions $\underline{\alpha}, \bar{\alpha}, \alpha \in \mathcal{K}_{\infty}$, and $\lambda, \sigma \in \mathcal{G}$, and for any triple of strictly positive real numbers $\left(\Delta_{x}, \Delta_{u}, \nu\right)$, there exists $T^{*}>0$ and for all $T \in\left(0, T^{*}\right)$ there exists a smooth function $V_{T}: \mathbb{R}^{n} \rightarrow$ $\mathbb{R}_{\geq 0}$ such that for all $|x| \leq \Delta_{x},|u| \leq \Delta_{u}$ the following holds:

$$
\begin{aligned}
\underline{\alpha}(|x|) \leq & V_{T}(x) \leq \bar{\alpha}(|x|) \\
V_{T}\left(F_{T}(x, u)\right)-V_{T}(x) \leq & -T \alpha(|x|)+T \lambda(|y|) \\
& +T \sigma(|u|)+T \nu .
\end{aligned}
$$


The function $V_{T}$ is called a SP-IOSS Lyapunov function. If the system is SP-IOSS with $\lambda=0$, we say that the system is semiglobally practically input to state stable (SP-ISS) and $V_{T}$ is called a SP-ISS Lyapunov function. If $\lambda=0$ and the system (1) is an input-free system $(\sigma=0)$, the system is semiglobally practically asymptotically stable (SP-AS) and $V_{T}$ is called a SP-AS Lyapunov function. Moreover, for SPISS, if the argument of $\alpha(\cdot)$ is the norm of the output $y$, which consists of only partial states, we have semiglobal practical quasi ISS (SP-qISS).

Defnition 2.2: [9] Let $\hat{T}>0$ be given and for each $T \in$ $(0, \hat{T})$ let the functions $V_{T}: \mathbb{R}^{n} \rightarrow \mathbb{R}_{\geq 0}$ and $u_{T}: \mathbb{R}^{n} \rightarrow \mathbb{R}$ be de£ned. We say that the pair $\left(u_{T}, V_{T}\right)$ is a semiglobally practically asymptotically (SPA) stabilizing pair for $F_{T}$ if there exist $\underline{\alpha}, \bar{\alpha}, \alpha \in \mathcal{K}_{\infty}$, such that for any pair of strictly positive real numbers $(\Delta, \nu)$ there exists a triple of strictly positive real numbers $\left(T^{*}, L, M\right)$, with $T^{*} \leq \hat{T}$, such that for all $x, z \in \mathbb{R}^{n}$ with $\max \{|x|,|z|\} \leq \Delta$, and $T \in\left(0, T^{*}\right)$ we have:

$$
\begin{aligned}
\underline{\alpha}(|x|) \leq V_{T}(x) & \leq \bar{\alpha}(|x|) \\
V_{T}\left(F_{T}(x, u)\right)-V_{T}(x) & \leq-T \alpha(|x|)+T \nu . \\
\left|V_{T}(x)-V_{T}(z)\right| & \leq L|x-z| \\
\left|u_{T}(x)\right| & \leq M .
\end{aligned}
$$

\section{Design tools}

\section{A. Framework for approximate based direct discrete-time design}

In this subsection we present a result from [7] on input to state stabilization via approximate discrete-time models. Consider a continuous-time nonlinear plant

$$
\dot{x}(t)=f(x(t), u(t), w(t)), \quad y(t)=h(x(t)),
$$

where $x \in \mathbb{R}^{n_{x}}, u \in \mathbb{R}^{m}, w \in \mathbb{R}^{p}$ and $y \in \mathbb{R}^{l}$ are respectively the state, control input, disturbance and output.

We assume that for any given $x_{0}, u(\cdot)$ and $w(\cdot)$ the differential equation in (8) has a unique solution defned on its maximal interval of existence $\left[0, t_{\max }\right)$. This may be guaranteed, for instance, by requiring $f$ in (8) to be locally Lipschitz. The control is taken to be a piecewise constant signal $u(t)=u(k T)=: u(k), \forall t \in[k T,(k+1) T), k \in \mathbb{N}$, where $T>0$ is the sampling period, and we suppose that the disturbance $w(\cdot)$ is constant during sampling intervals, that is $w(t)=w(k), \forall t \in[k T,(k+1) T)$. We assume that some combination (output) or all of the states $(x(k):=x(k T))$ are available at sampling instant $k T, k \in \mathbb{N}$. The exact discretetime model for the plant (8), which describes the plant behavior at sampling instants $k T$, is obtained by integrating the initial value problem

$$
\dot{x}(t)=f(x(t), u(k), w(t)),
$$

with given $w(k), u(k)$ and $x_{0}=x(k)$, over the sampling interval $[k T,(k+1) T]$. If we denote by $x(t)$ the solution of the initial value problem (9) at time $t$ with given $x_{0}=x(k)$, $u(k)$ and $w(k)$, then the exact discrete-time model of (8) can be written as:

$$
\begin{aligned}
x(k+1) & =x(k)+\int_{k T}^{(k+1) T} f(x(\tau), u(k), w(k)) d \tau \\
& =: F_{T}^{e}(x(k), u(k), w(k)) .
\end{aligned}
$$

Since $F_{T}^{e}$ is not known in most cases (see [7]), we use an approximate discrete-time model of the plant

$$
x(k+1)=F_{T}^{a}(x(k), u(k), w(k)) .
$$

to design a discrete-time controller for the original plant (8). For instance, the Euler approximate model is $x(k+1)=$ $x(k)+T f(x(k), u(k), w(k))$.

We consider a family of dynamic feedback controllers

$$
\begin{aligned}
z(k+1) & =G_{T}(x(k), z(k)) \\
u(k) & =u_{T}(x(k), z(k)),
\end{aligned}
$$

where $z \in \mathbb{R}^{n_{z}}$. We emphasize that if the controller (12) input to state stabilizes the approximate model (11) for all small $T$, this does not guarantee that the same controller would input to state stabilize the exact model (10) for all small $T$ (see [1], [2], [10]). The following result provides a framework for controller design via approximate discretetime models.

Theorem 3.1: [7] Suppose that there exist $\underline{\alpha}, \bar{\alpha}, \alpha \in \mathcal{K}_{\infty}$ and $\sigma \in \mathcal{K}$, and for any strictly positive real numbers $\left(\Delta_{1}, \Delta_{2}, \Delta_{3}, \nu\right)$ there exist $\varrho \in \mathcal{K}_{\infty}$, strictly positive real numbers $T^{*}, L, M$ such that for all $T \in\left(0, T^{*}\right)$ there exists a function $V_{T}: \mathbb{R}^{n_{x}+n_{z}} \rightarrow \mathbb{R}_{\geq 0}$ such that for all $|(x, z)| \leq \Delta_{1},|u| \leq \Delta_{2},|w| \leq \bar{\Delta}_{3}, T \in\left(0, T^{*}\right)$ we have: 1. SP-ISS Lyapunov conditions for closed-loop approximate; 2 . consistency between $F_{T}^{a}$ and $F_{T}^{e} ; 3$. uniform local boundedness of $u_{T}$ (see [7] for detail defnitions). Then, there exists $\beta \in \mathcal{K} L, \gamma \in \mathcal{G}$ such that for any strictly positive real numbers $\left(\widetilde{\Delta}_{1}, \widetilde{\Delta}_{2}, \widetilde{\nu}\right)$ there exists $\widetilde{T}>0$ such that for all $|(x(0), z(0))| \leq \widetilde{\Delta}_{1},\|w\|_{\infty} \leq \widetilde{\Delta}_{2}$ and $T \in(0, \widetilde{T})$ the solutions of (10), (12) satisfy SP-ISS of closed-loop exact.m

We emphasize that the consistency condition in Theorem 3.1 is checkable although $F_{T}^{e}$ is not known in general. Defnitions and lemmas that give suffcient conditions for consistency condition are stated in [7].

\section{B. Euler based discrete-time backstepping design}

In this subsection, a result from [9] is cited. The Euler model is used, since it preserves the strict feedback structure of the plant that is needed for a backstepping design and it satisfes the consistency property required by Theorem 3.1 .

Consider a continuous-time plant of the strict feedback form:

$$
\begin{aligned}
\dot{x} & =f(x)+g(x) \xi \\
\dot{\xi} & =u .
\end{aligned}
$$


The Euler approximate model of (13),(14) is:

$$
\begin{aligned}
& x(k+1)=x(k)+T(f(x(k))+g(x(k)) \xi(k)) \\
& \xi(k+1)=\xi(k)+T u(k) .
\end{aligned}
$$

Under certain properties and conditions (see [9]), there exists a SPA stabilizing pair $\left(u_{T}, V_{T}\right)$ for the Euler model (15),(16). In particular, we can take:

$$
u_{T}=-c\left(\xi-\alpha_{T}(x)\right)-\frac{\widetilde{\Delta W}_{T}}{T}+\frac{\Delta \alpha_{T}}{T},
$$

where $c>0$ is arbitrary, $\xi=\alpha_{T}(x)$ asymptotically stabilizes (13) and

$$
\begin{aligned}
\Delta \alpha_{T} & =\alpha_{T}(x+T(f+g \xi))-\alpha_{T}(x) \\
\widetilde{\Delta W_{T}} & = \begin{cases}\frac{\overline{\Delta W}_{T}}{\left(\xi-\alpha_{T}(x)\right)}, & \xi \neq \alpha_{T}(x) \\
T \frac{\partial W_{T}}{\partial x}(x+T(f+g \xi)) g, & \xi=\alpha_{T}(x)\end{cases} \\
\overline{\Delta W}_{T} & =W_{T}(x(k+1))-W_{T}\left(x+T\left(f+g \alpha_{T}\right)\right)
\end{aligned}
$$

and the Lyapunov function $V_{T}=W_{T}+\frac{1}{2}\left(\xi-\alpha_{T}(x)\right)^{2}$.

\section{A LaSalle criterion for SP-ISS}

The result from [6] on changing supply rates for SP-ISS discrete-time systems, provides a recipe for constructing a composite Lyapunov function to solve LaSalle type stability problem in sampled-data system. Consider the system (1). Using Corollary 5.1 of [6], we show that if the functions $V_{1 T}: \mathbb{R}^{n} \rightarrow \mathbb{R}_{\geq 0}$ and $V_{2 T}: \mathbb{R}^{n} \rightarrow \mathbb{R}_{\geq 0}$ are respectively a SP-qISS Lyapunov function and a SP-IOSS Lyapunov function of the system (1), and

$$
\lim \sup _{s \rightarrow+\infty} \frac{\lambda_{2}(s)}{\alpha_{1}(s)}<+\infty,
$$

Then, the function $V_{T}: \mathbb{R}^{n} \rightarrow \mathbb{R}_{\geq 0}$ that satis£es

$$
V_{T}=V_{1 T}+\rho\left(V_{2 T}\right) \text {. }
$$

where $\rho(s):=\int_{0}^{s} q(\tau) d \tau$, with $q \in \mathcal{S N}$ and $\rho \in \mathcal{K}_{\infty}$, is a SP-ISS Lyapunov function of the system (1).

\section{CONTROL OF AN ACTIVE SUSPENSION SYSTEM}

\section{A. Car suspension system modeling}

We use the quarter car model as the mathematical description of the suspension system, following the model used in [3]. The schematic diagram of the model is shown in Figure 1. In this model, the suspension actuator is taken to be a force actuator acting between the car body (the sprung mass) and the axle of the car. The tire is an ideal, undamped spring between the axle and the ground. Finally, the axle and wheel assemblies are represented as a mass (the unsprung mass) connected to the ground via the tire spring. As shown in Figure 1, the suspension force also reacts against the unsprung mass.

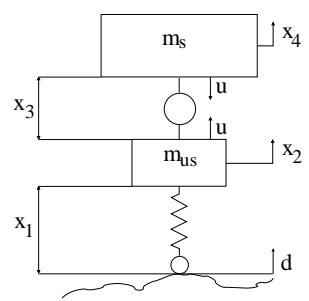

Fig. 1. The quarter car suspension model

A linear time invariant dynamic model of the system is represented as follows:

$$
\begin{array}{ll}
\dot{x}_{1}=x_{2}-d, & \dot{x}_{3}=-x_{2}+x_{4} \\
\dot{x}_{2}=-\omega^{2} x_{1}+\rho u, & \dot{x}_{4}=-u
\end{array}
$$

where $x_{1}$ - tire dexection $(\mathrm{m}), x_{2}$ - unsprung mass velocity (m/sec), $x_{3}$ - suspension dedection (m) and $x_{4}-$ sprung mass velocity $(\mathrm{m} / \mathrm{sec})$. The parameter $\omega$ is the unsprung mass natural frequency, $\rho$ is the sprung to unsprung mass ratio and assume that the travel limit of the suspension is $\pm D$. In other words, as long as the suspension dexection $x_{3}$ satisfes $-D<x_{3}<D$, the suspension will not bottom out. Following [3], we use the parameters $\omega=2 \pi \cdot 10 \mathrm{rad} / \mathrm{sec}$, $\rho:=m_{s} / m_{u s}=10, D=0.1 \mathrm{~m}$.

\section{B. Discrete-time backstepping controller design}

To obtain a strict feedback form, the state equations are reordered using the following diffeomorphism:

$$
\begin{aligned}
z_{1} & =x_{1}+\frac{\rho}{\rho+1} x_{3}, & z_{3} & =x_{3} \\
z_{2} & =\frac{1}{\rho+1} x_{2}+\frac{\rho}{\rho+1} x_{4}, & z_{4} & =-x_{2}+x_{4}
\end{aligned}
$$

The model is then rewritten in the following form

$$
\begin{aligned}
& \dot{z}_{1}=z_{2}-d \\
& \dot{z}_{2}=-\frac{\omega^{2}}{\rho+1} z_{1}+\frac{\rho \omega^{2}}{(\rho+1)^{2}} z_{3} \\
& \dot{z}_{3}=z_{4} \\
& \dot{z}_{4}=\omega^{2} z_{1}-\frac{\rho \omega^{2}}{\rho+1} z_{3}-(1+\rho) u=\tilde{u}
\end{aligned}
$$

The Euler model of the system in a strict feedback form is written as follow:

$$
\begin{aligned}
z_{1}(k+1)= & z_{1}(k)+T\left(z_{2}(k)-d\right) \\
z_{2}(k+1)= & z_{2}(k)+T\left(\frac{-\omega^{2} z_{1}(k)}{\rho+1}+\frac{\rho \omega^{2} z_{3}(k)}{(\rho+1)^{2}}\right) \\
z_{3}(k+1)= & z_{3}(k)+T z_{4}(k) \\
z_{4}(k+1)= & z_{4}(k)+T\left(\omega^{2} z_{1}(k)-\frac{\rho \omega^{2} z_{3}(k)}{\rho+1}\right. \\
& -(1+\rho) u(k))=z_{4}(k)+T \tilde{u}(k)
\end{aligned}
$$

In the design, the disturbance $d$ is taken to be zero, which is a reasonable approach since the disturbances affecting the system are nearly impulsive and thus correlate to nonzero 
initial conditions. Therefore, the problem is simplifed to an asymptotic stabilization problem. We follow similar design steps to those done in [3], applying the Euler based backstepping design [9] as cited in Subsection 3.2. Due to space limitation, some trivial steps are omitted.

Step 1: From the continuous-time model, it can be seen that if $z_{3} \equiv 0$, then subsystem (28), (29) is marginally stable. We design a virtual feedback control law $z_{3 d}\left(z_{1}, z_{2}\right)$ which is bounded between $-D$ and $D$ and renders the origin of the closed-loop $\left(z_{1}, z_{2}\right)$ subsystem SP-AS. A control that satisfes this is

$$
z_{3 d}=-D \tanh \left(\frac{k_{1} z_{2}}{D}\right), \quad k_{1}>0 .
$$

Unfortunately, the candidate Lyapunov function

$$
V_{0 T_{1}}\left(z_{1}, z_{2}\right)=\frac{1}{2} \frac{\omega^{2}}{\rho+1} z_{1}^{2}+\frac{1}{2} z_{2}^{2},
$$

which was used in the continuous-time design [3], gives

$$
\Delta V_{0 T_{1}} \leq-T M z_{2} \tanh \left(z_{2}\right)+T \nu_{01}
$$

with $z_{3}=z_{3 d}$, which is negative semidefnite with small offset $\nu_{01}>0$.

While we can apply LaSalle Invariance Principle for the continuous-time case, we cannot do the same for the sampled-data design when semiglobal stability condition occurs. The Euler based backstepping [9] we use does not facilitate this condition, and the candidate Lyapunov function $V_{0 T_{1}}$ does not satisfy the Erst condition of Theorem 3.1. To
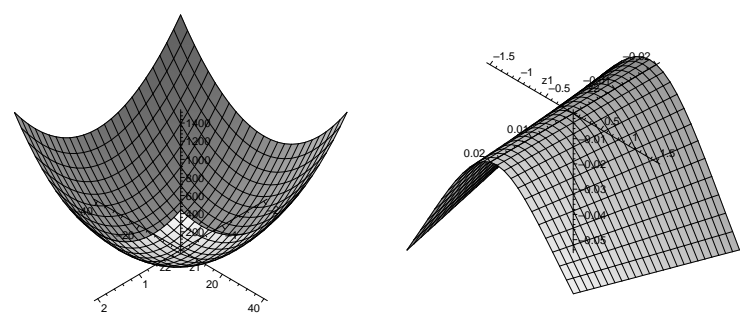

Fig. 2. Surface plots for $V_{0 T_{1}}$ (l) and $\Delta V_{0 T_{1}}$ (r), with $T=0.001 \mathrm{sec}$.

solve this problem, we apply Corollary 5.1 of [6] to construct a SP-AS Lyapunov function for subsystem (28), (29). It has been shown earlier that $V_{0 T_{1}}\left(z_{1}, z_{2}\right)$ is in fact a SP-qISS Lyapunov function for the subsystem. The surface plots of $V_{0 T_{1}}$ and $\Delta V_{0 T_{1}}$ are shown in Figure 2.

To show that the subsystem is SP-AS, we introduce another function

$$
V_{0 T_{2}}\left(z_{1}, z_{2}\right)=\frac{1}{2} \frac{\omega^{2}}{\rho+1} z_{1}^{2}+\frac{1}{2} z_{2}^{2}+\varepsilon \frac{z_{1} z_{2}}{\left(1+z_{1}^{2}\right)^{3 / 4}} .
$$

For small sampling period $T>0$ and small $\varepsilon>0$, the Lyapunov difference of $\Delta V_{0 T_{2}}$ satis£es

$$
\begin{aligned}
\Delta V_{0 T_{2}} \leq & -T M z_{2} \tanh \left(z_{2}\right)-T M_{1} z_{1}^{2} \\
& +T M_{3}\left(z_{2}^{2}+\tanh ^{2}\left(z_{2}\right)\right)+T \nu_{02}
\end{aligned}
$$

with some $M, M_{1}, M_{3}>0$. Hence, $V_{0 T_{2}}$ is a SP-IOSS Lyapunov function for the frst two subsystems.

From (34) and (36), it is obvious that the condition (21) is satisfed, and hence all conditions of Corollary 5.1 of [6] holds. Hence, we can conclude that for some $\rho \in \mathcal{K}_{\infty}$, the function $V_{0 T_{a}}$ that satis£es

$$
V_{0 T_{a}}=V_{0 T_{1}}+\rho\left(V_{0 T_{2}}\right)
$$

is a SP-AS Lyapunov function for the frst two subsystems. The surface plots of $V_{0 T_{2}}$ and $\Delta V_{0 T_{2}}$ are shown in Figure 3. Suppose we are given a set of initial conditions, such that the SP-AS property of the subsystem (28), (29) is guaranteed with $T=0.001 \mathrm{sec}$. For a $£ \mathrm{x} \varepsilon=0.1$, choosing an appropriate $\rho \in \mathcal{K}_{\infty}$, then we can use formula (37) to combine Figure 2 and Figure 3 after scaling $V_{0 T_{2}}$ with the function $\rho$, to show the SP-AS Lyapunov surface $V_{0 T_{a}}$ and the SP-AS difference $\Delta V_{0 T_{a}}$ of the subsystem (28), (29), for the given set of initial conditions. Choosing $\rho(\cdot)=\operatorname{Id}(\cdot)$


Fig. 3. Surface plots for $V_{0 T_{2}}$ (l) and $\Delta V_{0 T_{2}}$ (r), with $T=0.001 \mathrm{sec}$ and $\varepsilon=0.1$.

results in a Lyapunov function

$$
V_{0 T_{a}}=V_{0 T_{1}}+V_{0 T_{2}}
$$

for the subsystem (28), (29), and we can immediately see that with $z_{3}=z_{3 d}$ the Lyapunov difference $\Delta V_{0 T_{a}}=\Delta V_{0 T_{1}}+$ $\Delta V_{0 T_{2}}$ is negative defnite and hence the subsystem (28), (29) is SP-AS.

Remark 4.1: We emphasize that choosing $\rho=\mathrm{Id}$ in our case is possible since we are dealing with a semiglobal practical property. The $V_{0 T_{a}}$ obtained does not hold globally, because of the saturation coming from the tanh function in $z_{3 d}$. We also have to choose a small $\varepsilon$ to guarantee that $\Delta V_{0 T_{a}}$ does not become positive for quite large $z_{2}$.

To continue the design procedure, for the purpose of simpler computation, we choose to use

$$
V_{0 T}=\frac{1}{2} V_{0 T_{a}}=\frac{1}{2}\left(V_{0 T_{1}}+V_{0 T_{2}}\right),
$$

since it is allowable to scale a Lyapunov function with a constant. We also £x $\varepsilon=0.1$ in this design. It is obvious that $V_{0 T}$ satisfes the Erst condition of Theorem 3.1. 
Step 2: We defne $\xi_{1}=z_{3}-z_{3 d}\left(z_{2}\right)$ and denote $\xi_{2}:=\dot{\xi}_{1}$ to obtain the following third order Euler model:

$$
\begin{aligned}
& z_{1}(k+1)=z_{1}+T z_{2} \\
& z_{2}(k+1)=z_{2}+T\left(-\frac{\omega^{2}}{\rho+1} z_{1}+\frac{\rho \omega^{2}}{(\rho+1)^{2}}\left(z_{3 d}+\xi_{1}\right)\right) \\
& \xi_{1}(k+1)=\xi_{1}+T \xi_{2} .
\end{aligned}
$$

Using a candidate Lyapunov function

$$
V_{1 T}\left(z_{1}, z_{2}, \xi_{1}\right)=V_{0 T}\left(z_{1}, z_{2}\right)+k_{2} \frac{\xi_{1}^{2}}{2}, \quad k_{2}>0
$$

and choosing the stabilizing controller as

$$
\xi_{2 d}=-\frac{1}{k_{2}} \frac{\rho \omega^{2}}{(\rho+1)^{2}}\left(z_{2}+\frac{\varepsilon z_{1}}{\left(1+\left(z_{1}+T z_{2}\right)^{2}\right)^{3 / 4}}\right)-k_{3} \xi_{1},
$$

with $k_{3}>0$, we have

$$
\Delta V_{1 T}=\left.\Delta V_{0 T}\right|_{z_{3 d}}-T k_{2} k_{3} \xi_{1}^{2}+T \nu_{1},
$$

which is negative defnite with a small offset $\nu_{1}>0$. Hence, the equilibrium $\left(z_{1}, z_{2}, \xi_{1}\right)=(0,0,0)$ is SP-AS. Since $z_{3 d}(0)=0$ we can conclude that the origin $\left(z_{1}, z_{2}, z_{3}\right)=$ $(0,0,0)$ is also SP-AS.

Step 3: Backstepping $\xi_{2}$ through an integrator results in the dynamical system, whose Euler model can then be written as follow:

$$
\begin{aligned}
& z_{1}(k+1)=z_{1}+T z_{2} \\
& z_{2}(k+1)=z_{2}+T\left(-\frac{\omega^{2}}{\rho+1} z_{1}+\frac{\rho \omega^{2}}{(\rho+1)^{2}}\left(z_{3 d}+\xi_{1}\right)\right) \\
& \xi_{1}(k+1)=\xi_{1}+T \xi_{2} \\
& \xi_{2}(k+1)=\xi_{2}+T \tilde{\tilde{u}} .
\end{aligned}
$$

At this step, we consider a candidate Lyapunov function

$$
V_{2 T}\left(z_{1}, z_{2}, \xi_{1}, \xi_{2}\right)=V_{1 T}\left(z_{1}, z_{2}, \xi_{1}\right)+\frac{k_{4}}{2}\left(\xi_{2}-\xi_{2 d}\right)^{2},
$$

with $k_{4}>0$. We apply the formula (17) to obtain $\tilde{\tilde{u}}_{T}$ using the following terms:

$$
\begin{aligned}
\alpha_{T} & =\frac{-\rho \omega^{2}}{k_{2}(\rho+1)^{2}}\left(z_{2}+\frac{\varepsilon z_{1}}{\left(1+\left(z_{1}+T z_{2}\right)^{2}\right)^{3 / 4}}\right)-k_{3} \xi_{1} \\
W_{T} & =\frac{k_{2}}{2 k_{4}} \xi_{1}^{2},
\end{aligned}
$$

(it turns out that $\alpha_{T}:=\xi_{2 d}$ ) and get

$$
\begin{aligned}
\frac{\Delta \alpha_{T}}{T} & =\frac{\xi_{2 d}(k+1)-\xi_{2 d}(k)}{T} \\
= & -\frac{1}{k_{2}} \frac{\rho \omega^{2}}{(\rho+1)^{2}}\left(\zeta_{2}+\frac{\varepsilon z_{1}}{\left(1+\left(z_{1}+2 T z_{2}+T^{2} \zeta_{2}\right)^{2}\right)^{3 / 4}}\right) \\
& -\frac{1}{k_{2} T} \frac{\rho \omega^{2}}{(\rho+1)^{2}} \varepsilon z_{1}\left(\frac{1}{\left(1+\left(z_{1}+2 T z_{2}+T^{2} \zeta_{2}\right)^{2}\right)^{3 / 4}}\right. \\
& \left.-\frac{1}{\left(1+\left(z_{1}+T z_{2}\right)^{2}\right)^{3 / 4}}\right)-k_{3} z_{4} \\
& -\frac{1}{T} k_{3} D\left(\tanh \left(z_{2}+T \zeta_{2}\right)-\tanh \left(z_{2}\right)\right),
\end{aligned}
$$

where $\zeta_{2}:=-\frac{\omega^{2}}{\rho+1} z_{1}+\frac{\rho \omega^{2}}{(\rho+1)^{2}} z_{3}$, and

$$
\begin{aligned}
\overline{\Delta W}_{T} & =V_{1 T}^{+}\left(\xi_{1}+T \xi_{2}\right)-V_{1 T}^{+}\left(\xi_{1}+T \xi_{2 d}\right) \\
& =\frac{k_{2}}{2 k_{4}}\left(2 T \xi_{1} \xi_{2}-2 T \xi_{1} \xi_{2 d}+T^{2} \xi_{2}^{2}-T^{2} \xi_{2 d}^{2}\right) .
\end{aligned}
$$

Moreover, using (19) we have

$$
\frac{\widetilde{\Delta W}_{T}}{T}=\frac{k_{2}}{k_{4}} \xi_{1}+\frac{k_{2} T}{2 k_{4}}\left(\xi_{2}+\xi_{2 d}\right) .
$$

Hence, we obtain $\tilde{\tilde{u}}=\tilde{\tilde{u}}_{T}$ by substituting (44),(45) to

$$
\tilde{\tilde{u}}_{T}=-\frac{k_{5}}{k_{4}}\left(\xi_{2}-\xi_{2 d}\right)-\frac{\widetilde{\Delta W_{T}}}{T}+\frac{\Delta \alpha_{T}}{T}, \quad k_{5}>0 .
$$

It can be shown that implementing $\tilde{\tilde{u}}_{T}$ to the system results in $\Delta V_{2 T}$ negative defnite with small offset $\nu_{2}>0$. This means that the equilibrium $\left(z_{1}, z_{2}, \xi_{1}, \xi_{2}\right)=(0,0,0,0)$ is SP-AS. Since $z_{3 d}(0)=0$, then the origin $\left(z_{1}, z_{2}, z_{3}, z_{4}\right)=(0,0,0,0)$ is also SP-AS.

We have seen earlier that $V_{1 T}$ satis£es the SP-ISS (in this case SP-AS) Lyapunov condition of Theorem 3.1. It is then obvious that with $V_{2 T}$ that the £rst condition of Theorem 3.1 still holds.

Step 4: Following exactly as in the continuous-time design, the resulting control law $u=u_{T}$ that SPA stabilizes the Euler model (28)-(31) has form

$$
u_{T}=\frac{1}{\rho+1}\left(-\tilde{\tilde{u}}_{T}-\Delta \zeta_{3 d}+\omega^{2} x_{1}\right)
$$

with $\Delta \zeta_{3 d}:=\ddot{z}_{3 d}$. Finally, by substituting the appropriate terms, we have $u_{T}$ as a nonlinear control law parameterized by the sampling period $T$ and £ve positive tuning parameters $k_{1}, k_{2}, k_{3}, k_{4}$ and $k_{5}$.

Expanding $u_{T}$ in series representation, we can show that $u_{T}$ satisfes the third condition of Theorem 3.1. Since all conditions of Theorem 3.1 hold, we can guarantee that $u_{T}$ SPA stabilizes the closed-loop approximate model, and also stabilize the closed-loop exact model. We further use results from [8], to conclude the SP-AS for the sampled data system (23), (47).

\section{Comparing the Euler-based controller with the Emula- tion controller}

We have designed a discrete-time backstepping controller (47) that SPA stabilizes the active suspension system. Now, we implement the controller (47), and observe the performance of the closed-loop sampled-data system with the designed controller, and compare it with a controller that has form:

$$
u=\frac{1}{\rho+1}\left(-\tilde{\tilde{u}}-\Delta \zeta_{3 d}+\omega^{2} x_{1}\right)
$$

where $\tilde{\tilde{u}}=\Delta \xi_{2 d}-\frac{k_{2} \xi_{1}}{k_{4}}-\frac{k_{5}}{k_{4}}\left(\xi_{2}-\xi_{2 d}\right)$, with $\Delta \xi_{2 d}:=\dot{\xi}_{2 d}$. The controller (48) is obtained via emulation design, by holding the continuous control constant during every sampling 
period (using zero order hold). By applying Corollary 5.1 of [5], we can show that the discrete-time emulation controller (48) also SPA stabilizes the continuous-time plant (23).

We study the condition when there are small offsets to the initial states, in other words, when allowing nonzero initial states. We observe the responses of the system to bumps of different heights and compare the performance of each controller. The shape of the isolated bump ${ }^{1}$ is chosen to be in the form that gives rise to the following velocity input:

$$
d(t)= \begin{cases}0, & t \leq 0 \\ 10 \pi A \sin (20 \pi t), & 0<t \leq 0.1 \\ 0, & t>0.1\end{cases}
$$

We frst run the Simulation Road-1 (see Figure 4), when setting $T=0.001 \mathrm{sec}$, initial states $\left(\begin{array}{llll}0.01 & 0 & 0.01 & 0\end{array}\right)^{T}$ and bump height $A=0.01 \mathrm{~m}$.
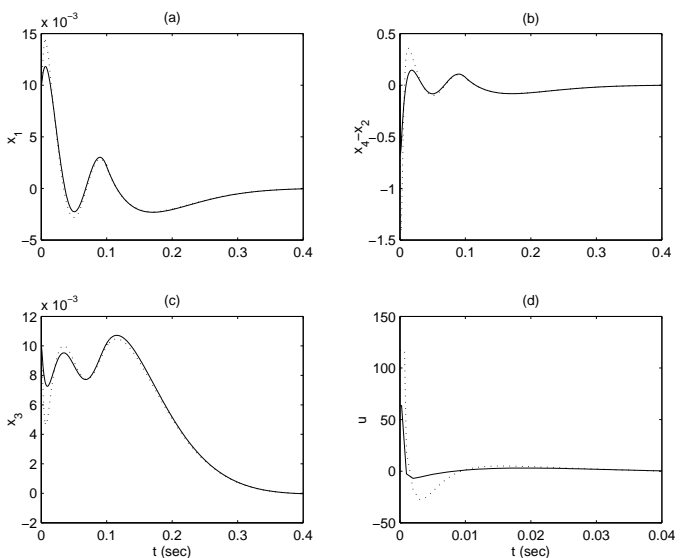

Fig. 4. Simulation Road-1 for a low bump condition, $\cdots$ emulation and --- Euler.

In Simulation Road-2 (see Figure 5), we set $A=0.1$ meter, which is considered as a high bump. We set $T=0.001$ sec and initial states $\left(\begin{array}{llll}0.01 & 0 & 0.01 & 0\end{array}\right)^{T}$.

From the two sets of simulation, we see that the system can always achieve better performance with the Euler based controller than with the emulation controller.

\section{REFERENCES}

[1] J.P. Barbot and S. Monaco and D. Normand-Cyrot, A sampled normal form for feedback linearization, Math. Contr. Sig. Sys., vol. 9, 1996, pp 162-188.

[2] F. Esfandiari and H.K. Khalil, On the robustness of sampled-data control to unmodelled high frequency dynamics, IEEE TAC, vol. 34, 1989, pp 900-903.

[3] N. Karlsson, A. Teel and D. Hrovat, "A backstepping approach to control of active suspensions", Proc. IEEE CDC, Orlando, FL, 2001, pp 4170-4175.

\footnotetext{
${ }^{1}$ this kind of bump is a haversine of height $A \mathrm{~m}$ and length $l=2 \mathrm{~m}$, while assuming the vehicle is traversing the road at a speed of $v=20 \mathrm{~m} / \mathrm{s}$.
}
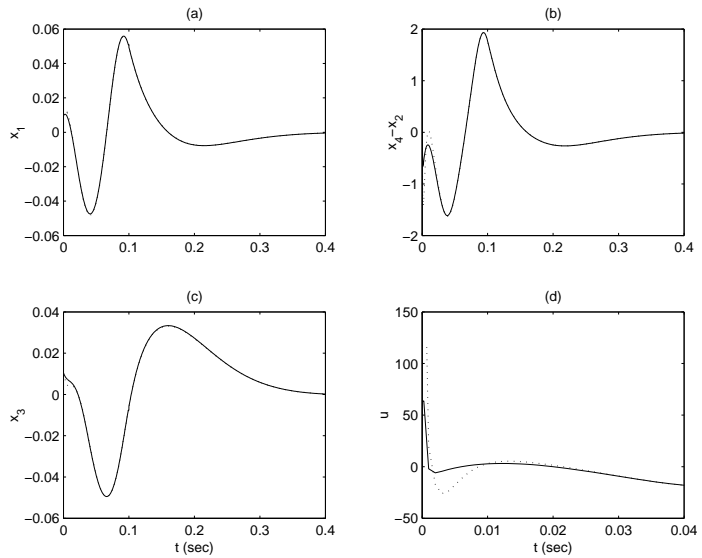

Fig. 5. Simulation Road-2 for a high bump condition, $\cdots$ emulation and --- Euler.

[4] M. Krstić, I. Kanellakopoulos and P. V. Kokotović, Nonlinear and Adaptive Control Design, Wiley; 1995.

[5] D.S. Laila, D. Neß̉ić and A.R. Teel, Open and closed loop dissipation inequalities under sampling and controller emulation, European Journal of Control, vol. 8, 2002, pp 109-125.

[6] D.S. Laila and D. Neşić, Changing supply rates for input-output to state stable discrete-time nonlinear systems with applications, Automatica, vol. 39, 2003, pp 821-835.

[7] D. Neß̧ić and D.S. Laila, A note on input to state stabilization for nonlinear sampled-data systems, IEEE TAC., vol. 47, 2002, 1153-1158.

[8] D. Nesić A.R. Teel and E.D. Sontag, Formulas relating $\mathcal{K} \mathcal{L}$ stability estimates of discrete-time and sampleddata nonlinear systems, $S C L$, vol. 38, 1999, pp 49-60.

[9] D. Nešić and A.R. Teel, "Backstepping on the Euler approximate model for stabilization of sampled-data nonlinear systems", Proc. IEEE CDC, Orlando, FL, 2001, pp 1737-1742.

[10] D. Nešić and A.R. Teel and P. Kokotović, Suffcient conditions for stabilization of sampled-data nonlinear systems via discrete-time approximations, $S C L$, vol. 38, 1999, pp 259-270. 